\title{
N AND FE-PEAK ELEMENTAL ABUNDANCES FROM IUE CO- ADDED SPECTRA OF HG-MN AND NORMAL STARS
}

\author{
SCOTT W. ROBY \\ Dept. of Phys. and Astr., Univ. of DE, Newark, DE 19716, USA (On \\ leave from Dept. of Earth Sci., SUNY College, Oswego, NY 13126, USA) \\ SAUL J. ADELMAN \\ Dept. of Physics, The Citadel, Charleston, SC 29409, USA \\ DAVID S.LECKRONE \\ NASA-Goddard Space Flight Center, Greenbelt, MD 20771, USA \\ CHARLES COWLEY \\ Astronomy Dept., Univ. of Michigan, Ann Arbor, MI 48109, USA \\ GLENN M. WAHLGREN \\ CSC/GHRS, Code 680, GSFC, Greenbelt, MD, 20771, USA
}

\begin{abstract}
Leckrone and Adelman have established an IUE observing strategy that has yielded co-added spectra with enhanced $\mathrm{S} / \mathrm{N}$ ratios for several A and B stars. New observations by Roby and Adelman using the same technique have added two new $\mathrm{Hg}-\mathrm{Mn}$ stars into this sample. We have begun a long-term study of elemental abundances in this uniform, high-quality set of IUE spectra for 13 stars. We report on the first stages of this project: abundances for $\mathrm{N}, \mathrm{Cr}, \mathrm{Mn}, \mathrm{Fe}, \mathrm{Co}$, and $\mathrm{Ni}$. The study of the Fe- peak elements show that our data set can provide accurate abundances and that abundances obtained from UV and optical spectra often are in good agreement. This study provides the groundwork for selfconsistent abundance analyses of more exotic elements in our long term project.
\end{abstract}

\section{INTRODUCTION}

We have begun a long term project using a homogenously observed set of high quality co-added IUE spectra for late B and early A main-sequence stars. The primary goal is to perform a self-consistent UV and optical analysis of the chemical compositon of these stars. The first step in the UV portion of the project is a determination of $\mathrm{Fe}$-peak $(\mathrm{Cr}, \mathrm{Mn}, \mathrm{Fe}, \mathrm{Ni}$, and $\mathrm{Co}$ ) elemental abundances to check the accuracy of our techniques and data and to provide a foundation for removing the blends due to these elements when studying other species (C, O, Al, P, S, Cl, $\mathrm{Cu}$ and $\mathrm{Zn}$ ) later on. We report here on this 
first phase of the project, which is nearing completion. We also report on two new N I abundances determined from two recently observed $\mathrm{Hg}-\mathrm{Mn}$ stars. The details of this separate study are reported elsewhere (Roby, 1990; Roby, Leckrone, and Adelman, 1992).

\section{OBSERVATIONS}

The IUE telescope is ideally suited to this program in that extensive wavelength coverage is needed with at least moderate resolution to sample the largest number of UV transitions possible for each species. Leckrone and Adelman (1989) have acheived excellent (for IUE) S/N ratios of 25 - 50 by a suitable strategy of co-adding multiple spectra of the same target using different exposure times (to cover the dynamic range of the detector) and different offsets along the dispersion axis (to reduce fixed pattern noise). Roby and Adelman recently observed two more $\mathrm{Hg}-\mathrm{Mn}$ stars using the same observing strategy. The program now contains SWP and LWP/LWR co-added images for eight normal stars, four $\mathrm{Hg}-\mathrm{Mn}$ stars and one horizontal-branch field star. At this time, the Fe-peak analysis has been completed for five normal stars and the four Hg-Mn stars utilizng the LWP/LWR co-added spectra.

\section{ANALYSIS}

\section{Adopted Parameters}

The model atmospheres used were generated by Adelman using the ATLAS program and are based on his optical abundance studies of $v$ Her (Adelman, 1992), $\pi$ Cet, $21 \mathrm{Aql}$, and $\nu$ Cap (Adelman, 1991), ¿ CrB (Adelman, 1989), $\mu$ Lep, $\kappa$ Cnc, $\theta$ Leo, and $o$ Peg (Adelman, 1988). The adopted atmospheric parameters are shown in Table 1.

TABLE I Atmospheric Parameters

\begin{tabular}{lrrlrr}
\hline star & $T_{\text {eff }}(\mathrm{K})$ & $\log \mathrm{g}$ & metallicity & $\xi k m \mathrm{~s}^{-1}$ & $\mathrm{v} \sin \mathrm{i}$ \\
\hline$\pi$ Cet & 13150 & 3.65 & opt. abund. & 0 & 18 \\
21 Aql & 12900 & 3.35 & opt. abund. & 0 & 19 \\
$\nu$ Cap & 10250 & 3.90 & opt. abund. & 0 & 17 \\
$o$ Peg & 9600 & 3.60 & opt. abund. & 1.7 & 9 \\
$\theta$ Leo & 9250 & 3.55 & opt. abund. & 1.8 & 18 \\
& & & & & \\
$\kappa$ Cnc & 13125 & 3.45 & opt. abund. & 0 & 6 \\
$\mu$ Lep & 12500 & 3.50 & opt. abund. & 0 & 18 \\
$v$ Her & 11900 & 3.60 & opt. abund. & 0 & 7 \\
$\iota$ CrB & 11250 & 3.65 & opt. abund. & 0.2 & 3 \\
\hline \hline
\end{tabular}

The atomic data used for computing UV synthetic spectra are contained in Kurucz's extensive linelist (Kurucz and Peytremann, 1975; Kurucz, 1981, 
1992), which include new calculations for the first nine ionization states of elements $\mathrm{z}=20-28$. Optical abundances from the previously cited studies of Adelman were used as the initial input for the synthetic spectra. For C, N, and $O$, the values of Roby and Lambert (1990) were used.

\section{Continuum Fitting}

The IUE/RDAF interactive normalization procedure NORM was modified by Roby to accommodate a scrolling graphic window so that a $500 \AA$ chunk of a high dispersion IUE spectrum can be scrolled horizontally with the user seeing only $20 \AA$ or so at a time. NORM was further modified to allow up to 150 points to be specified in normalizing these large chunks. Typically 100 points were required to fit a typical $500 \AA$ chunk. For late B and early A mainsequence stars, the high peaks in the observed spectrum very often lie $1-5 \%$ below the true continuum. Thus it is necessary to do some modeling of the spectrum to determine the location of the continuum. For these calculations we adopted the initial parameters described in the previous section.

A major concern is that the abundances adopted (particularly Fe) for determining the continuum placement may effect the final abundance determinations. Fortunately, we have some evidence that the optical Fe abundance should be reliable in fitting the continuum. Two of us (Leckrone and Wahlgren) have observed $\chi$ Lup and $\kappa$ Cnc with the Goddard High Resolution Spectrograph (GHRS) on the HST. The high resolution of these two sharp-lined spectra reveal the continuum quite readily. Analyses of this data shows that the optical Fe abundance requires adjustments of less than 0.1 dex in the log to model the GHRS spectra successfully. Our experience with IUE data shows that a 0.1 dex change in $\log \mathrm{Fe} / \mathrm{H}$ changes the location of the continuum with respect to high points in the spectrum by less than $1 \%$. This is within our estimated range of internal accuracy $(0.5-2.0 \%)$ in fitting the continuum with models.

Each coadded spectrum was given a trial normalization based on the high peaks found in the observed spectrum with the RDAF procedure NORM. This was then plotted along with an overlapping synthetic spectrum based on optical abundances. The original $500 \AA$ observed spectrum chunk was then normalized anew using this plot as a guide. When possible several lines over a small region were used to judge the placement of the continuum - in other cases a single feature or two dominates the only usable peak and a subjective judgment had to be made. A linear interpolation of the fitted continuum was then divided into the observed flux to get the final normalized flux used for subsequent abundance determinations. The continuum fit division also removes what appears to be residual echelle ripples in the data. Examples of the final normalized continua are shown in Figure 1.

Subsequent reviews of the continuum fit during the abundance determinations of individual lines revealed that the above technique yielded a continuum fit judged to be good about $95 \%$ of the time. In the few bad cases, the continuum was readjusted locally. 


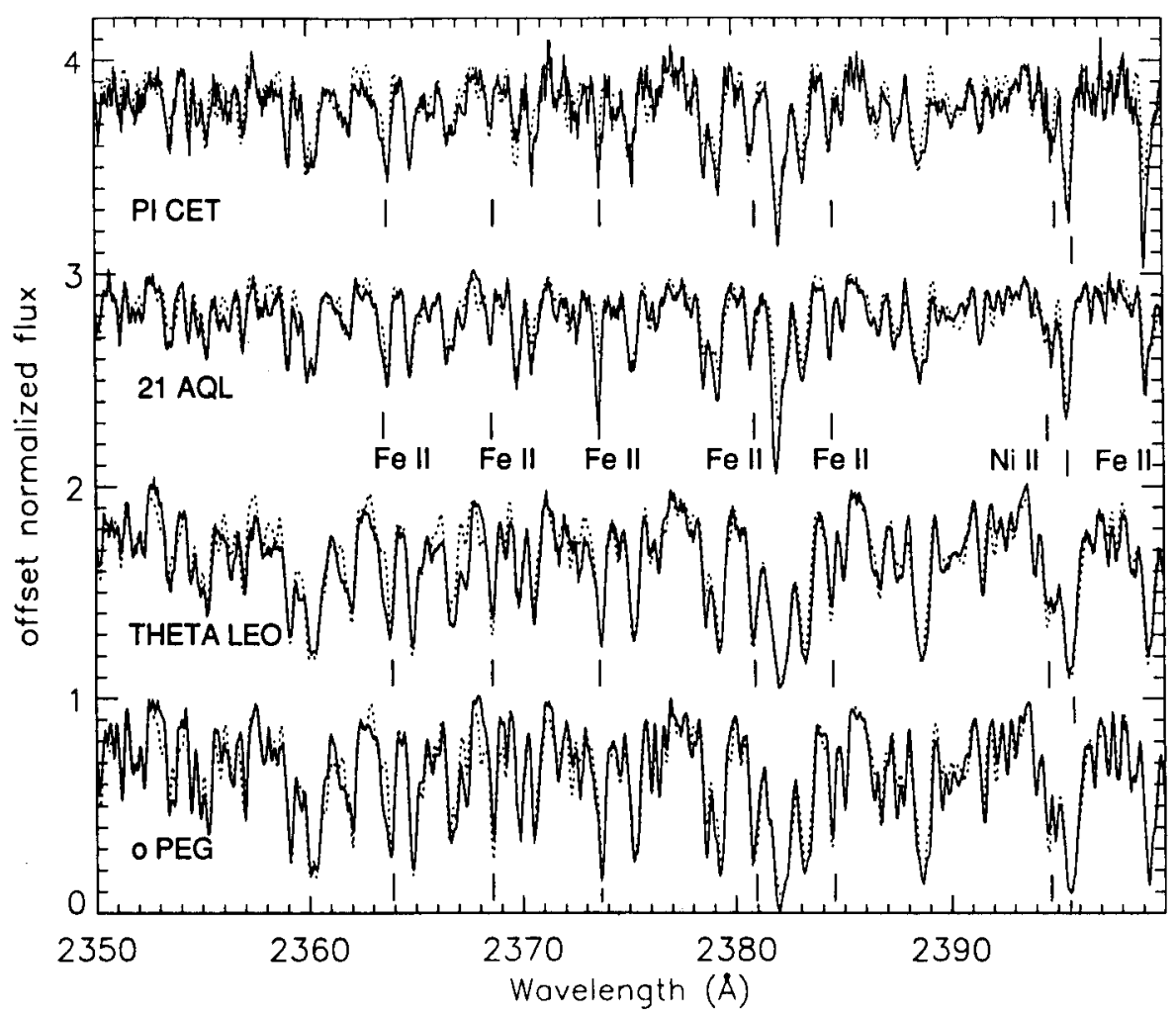

FIGURE 1: SAMPLE OF CONTINUUM FITS IN FOUR STARS. Each star is vertically offeet by addition of $0,1,2$, or 3 to the normalized flux. The solid lines show the IUE co-added observations; dotted lines show synthetic spectra (based on optical abundances) used for modelling the continuum. Lines used in our abundance analyses are denoted by vertical bars. Note the general agreement between initial optical-based models and observations. Also note that a single Fe abundance will not fit each Fe II line in a given star.

Line Analysis

To choose the least-blended lines for our analysis, we computed global synthetic spectra for the range $2000-3000 \AA$ in the $\mathrm{Hg}-\mathrm{Mn}$ star, $\kappa$ Cnc. For each of six elements ( $\mathrm{Ti}, \mathrm{Cr}, \mathrm{Mn}, \mathrm{Fe}, \mathrm{Ni}$, and $\mathrm{Co}$ ) we also calculated spectra with their abundance set effectively to zero. Dividing these into the full element spectrum produces a synthetic spectrum showing only the lines of the chosen element (see Fig. 2). Thus, at a glance, we could quickly see which features involved blends of different species and which did not. We further examined sources of $g f$ values, especially the compilations by Martin, Fuhr, and Wiese (1988) and Fuhr, Martin, and Wiese (1988), as we desired to use lines with the best quality $g f$ values. We also used the completeness and symmetry of the line profile and the location of local continuum points as additional criteria in selecting lines. Abundances for indivdual lines were obtained by examining plots with overlaying synthetic spectra that bracketed the observed profile in strength. Two independent judgements were made for each line (by Roby and 
Adelman) of the abundance needed to make a fit. We usually agreed to within 0.05 dex, conversing by mail.

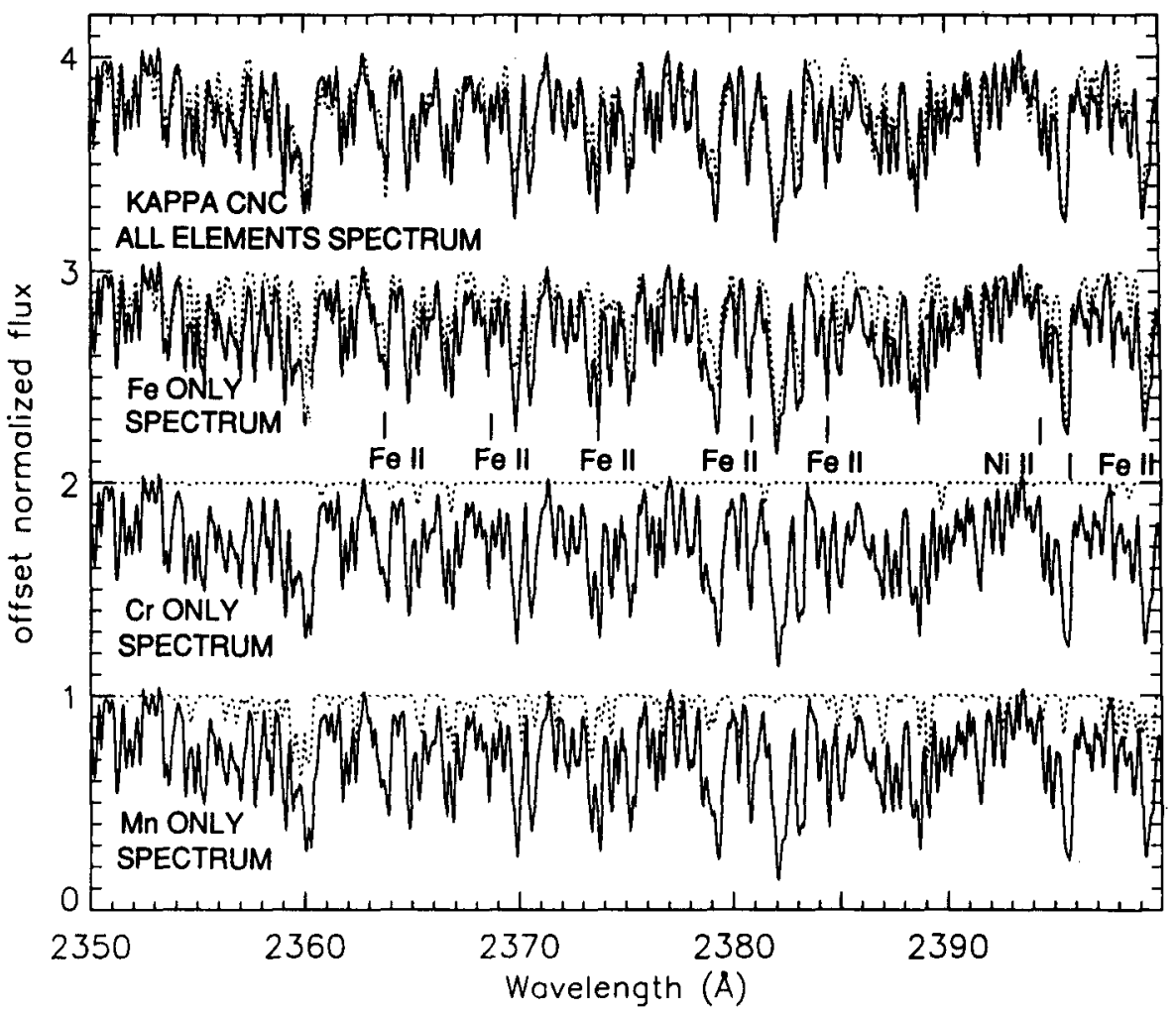

FIGURE 2: A NOVEL APPROACH TO IDENTIFYING LINE BLENDS. Vertical offects by addition of 0, 1, 2, or 3 used. Solid lines show the IUE co-added observation of $x$ Cnc; dotted lines show synthetic spectra. The oynthetic spectrum labeled "All Elements Spectrum" is calculated with all elements using optical abundances. The "Fe Only Spectrum" is a synthetic spectrum showing only the Fe lines (see text). Similar single-element spectra were calculated for $\mathrm{Cr}, \mathrm{Mn}$ (both shown here), Ni, Co, and Ti from 1150 to $3000 \mathrm{~A}$.

\section{RESULTS}

The abundance determinations are shown in Tables II and III. The abundances are relative to $\log \mathrm{H}=0$. The internal rms scatter (std) of the individual abundances for the lines of each species is shown in parentheses, followed by the number of lines $(n)$ used. For N I, the std and $n$ columns have been left out to save space $(\mathrm{n}=3$ and std is typically $(0.35))$.

The internal scatter for individual lines of $\mathrm{Fe}$ II and $\mathrm{Cr}$ II are in the range $0.1-0.2$ dex, and slightly larger for $\mathrm{Ni}$ II. Comparison with the optical 
abundances for these three species usually show a difference that is the same or smaller than the internal scatter. In the worst cases the difference is about two standard deviations.

TABLE II Cr II, Fe II, and Ni II Log N/H Abundances

\begin{tabular}{|c|c|c|c|c|c|c|c|c|c|}
\hline star & Cr II & std & $\mathrm{n}$ & FE II & std & $\mathrm{n}$ & $\mathrm{Ni} I I$ & std & $\mathrm{n}$ \\
\hline$\pi$ Cet & -6.09 & $(0.17)$ & 5 & -4.61 & $(0.16)$ & 25 & -5.80 & $(0.24)$ & 6 \\
\hline $21 \mathrm{Aql}$ & -6.58 & $(0.24)$ & 7 & -4.69 & $(0.29)$ & 22 & -6.07 & $(0.32)$ & 9 \\
\hline$\nu$ Cap & -5.72 & $(0.24)$ & 7 & -4.57 & $(0.09)$ & 24 & -5.40 & $(0.12)$ & 9 \\
\hline$o \mathrm{Peg}$ & -6.20 & $(0.19)$ & 7 & -4.62 & $(0.11)$ & 24 & -5.17 & $(0.15)$ & 8 \\
\hline$\theta$ Leo & -6.08 & $(0.23)$ & 7 & -4.82 & $(0.09)$ & 26 & -5.40 & $(0.22)$ & 8 \\
\hline$\kappa \mathrm{Cnc}$ & -6.28 & $(0.22)$ & 7 & -4.53 & $(0.17)$ & 26 & -5.87 & $(0.22)$ & 9 \\
\hline$\mu$ Lep & -5.64 & $(0.19)$ & 7 & -4.79 & $(0.12)$ & 26 & -6.33 & $(0.50)$ & 8 \\
\hline$v \mathrm{Her}$ & -5.91 & $(0.24)$ & 6 & -4.92 & $(0.15)$ & 25 & -6.19 & $(0.29)$ & 9 \\
\hline$\iota \mathrm{CrB}$ & -5.95 & $(0.19)$ & 6 & -4.46 & $(0.12)$ & 25 & -6.04 & $(0.26)$ & 9 \\
\hline
\end{tabular}

TABLE III Mn II, Mn III, Co II, and N I Log N/H Abundances

\begin{tabular}{lcccccccccc}
\hline star & Mn II & std & n & Mn III & std & n & Co II & std & n & N I \\
\hline$\pi$ Cet & -5.97 & $(0.23)$ & 8 & -5.38 & $(0.21)$ & 2 & -6.54 & $(0.53)$ & 3 & -4.70 \\
21 Aql & -6.23 & $(0.22)$ & 9 & -6.46 & $(0.07)$ & 2 & -6.84 & $(0.71)$ & 4 & -4.45 \\
$\nu$ Cap & -5.90 & $(0.25)$ & 5 & - & - & - & -6.94 & $(0.32)$ & 2 & -4.92 \\
$o$ Peg & -6.20 & $(0.23)$ & 8 & - & - & - & -6.37 & $(0.17)$ & 4 & -4.20 \\
$\theta$ Leo & -6.31 & $(0.25)$ & 7 & - & - & - & -6.91 & $(0.40)$ & 4 & -4.60 \\
& & & & & & & & & & \\
$\kappa$ Cnc & -3.98 & $(0.32)$ & 9 & -3.65 & $(0.27)$ & 7 & $<-7.36 ?$ & $(0.77)$ & 4 & $<-6.12$ \\
$\mu$ Lep & -4.29 & $(0.24)$ & 8 & -3.68 & $(0.17)$ & 5 & $<-8.31 ?$ & $(0.44)$ & 3 & $<-6.19$ \\
$v$ Her & -4.44 & $(0.15)$ & 8 & -4.00 & $(0.40)$ & 5 & $<-8.16 ?$ & $(0.50)$ & 4 & $<-6.43$ \\
$\iota$ CrB & -5.00 & $(0.28)$ & 8 & -4.58 & $(0.44)$ & 4 & $<-8.06 ?$ & $(0.72)$ & 4 & $<-6.55$ \\
\hline \hline
\end{tabular}

For the two normal stars ( $o$ Peg and $\theta$ Leo) where $\mathrm{Mn} \mathrm{II} \mathrm{and} \mathrm{Co} \mathrm{I} \mathrm{have} \mathrm{been}$ reliably measured in the optical, we find good agreement with the UV results. However, in the Hg-Mn stars, the UV abundance is larger $(+0.30$ dex on average) than the optical abundance for Mn II. This small discrepancy is perhaps related to the enhanced strength of $\mathrm{Mn}$ II lines in these stars and may be a signature of non-LTE effects, inadequate knowledge of the atomic parameters, or additional contributions from unaccounted for weaker lines in the UV spectrum.

Also in the Hg-Mn stars, we find a very large internal scatter for the individual line abundances of $\mathrm{Mn}$ III and Co II. We suspect that unaccounted for line blends are primarily responsible, as these lines are very weak, and therefore more susceptible to contributions from weak, unidentified lines. The 
lack of detection of some of the Co II lines while others in the same star appear much stronger than the detection limit requires further attention. We also note that the Mn III lines yield abundances systematically higher (about 0.45 dex) than the Mn II lines in the same stars. This also may be a signature of nonLTE.

The N I results for $\mu$ Lep and $v$ Her are reported here for the first time. They are upper limits, but more confining than those provided by near-IR studies, due to the larger intrinsic strength of the UV lines. All four $\mathrm{Hg}-\mathrm{Mn}$ stars consistently suggest that the N I abundance is extremely depleted, by factors of 40 - 100 relative to the normal stars (4-10 times more depleted than indicated by less stringent near-IR upper limits).

The implications of these results will be discussed in forthcoming papers (Fe-peak abundances; Adelman et al. , 1992, N I; Roby et al. , 1992).

\section{REFERENCES}

Adelman, S. J. 1988, M.N.R.A.S., 235, 749.

Adelman, S. J. 1989, M.N.R.A.S., 239, 487.

Adelman, S. J. 1991, M.N.R.A.S., 252, 116.

Adelman, S. J. 1992, M.N.R.A.S., in press.

Adelman, S. J., Roby, S. W., Leckrone, D. s., Cowley, C., and Wahlgren, G. M. 1992 , in preparation.

Fuhr, J. R., Martin, G. A., and Wiese, W. L. 1988, Journal of Physical and Reference Data, 17, Suppl. 4.

Leckrone, D. S., and Adelman, S. J. 1989, Ap. J. Suppl., 71, 387.

Kurucz, R. L. 1981, SAO Special Report 390 .

Kurucz, R. L. 1992, private communication.

Kurucz, R. L., and Peytremann, E. 1975, SAO Special Report 367.

Martin, G. A., Fuhr, J. R. and Wiese, W. L. 1988, Journal of Physical and Reference Data, 17, Suppl. 3.

Roby, S. W., and Lambert, D. L. 1990, Ap. J. Suppl., , 73, 167.

Roby, S. W., Leckrone, D. S., and Adelman, S. J. 1992, in preparation.

Roby, S. W. 1990, in Evolution of Stars: The Photospheric Abundance

Connection; Poster Paper Supplement, ed G. Michaud, A. Tutukov, and M. Bergevin, 145th I.A.U. Symposium, p. 75. 